available. Our local results are promising but larger series with long-term follow-up are required to determine its superiority.

Disclosures B. Pabon Guerrero: None. M. Patino Hoyos: None. J. Gutierrez Banos: None. V. Torres: None. O. Vargas: None. L. Moreira Ponce: None. J. Mejia: None.

\section{0-016 SURPASS EMBOLIZATION OF INTRACRANIAL ANEURYSMS AT TWO HIGH VOLUME COMPREHENSIVE STROKE CENTERS: UNEXPECTEDLY HIGH RATE OF NEUROLOGIC COMPLICATIONS}

${ }^{1} S$ Roychowdhury* ${ }^{2}$ I Kane, ${ }^{2} \mathrm{~A}$ Saifuddin, ${ }^{3} \mathrm{P}$ Khandelwal, ${ }^{1} \mathrm{E}$ Nourallah-Zadeh, ${ }^{4} \mathrm{G}$ ${ }^{1}$ Department of Radiology and Neurosurgery, University Radiology Group, East Brunswick, NJ; ${ }^{2}$ Radiology, Rutgers Robert Wood Johnson Medical School, New Brunswick, NJ; ${ }^{3}$ Endovascular Neurological Surgery and Neurology, University Hospital, Newark, NJ; ${ }^{4}$ Department of Neurosurgery, University Radiology Group, New Brunswick, NJ

10.1136/neurintsurg-2020-SNIS.16

Introduction/Purpose The Surpass flow-diverter (FD) has been offered as a new alternative to preexisting Pipeline FD with a comparable safety profile. ${ }^{i}$ Prior publications have listed neurologic morbidity and mortality complication rates as $6 \%$ and $2.7 \%$ for the Surpass FD \& $8.4 \%$ \& $3.8 \%$ for the Pipeline FD respectively. ${ }^{1,2,3}$ Anecdotal national experiences with the Surpass FD have been mixed, and there is a scarcity of data on real world complications of Surpass FD. A systematic review of cases performed at a high volume comprehensive stroke center involving the Surpass device was conducted.

Materials and Methods A review of all aneurysms treated with the Surpass FD at two high volume aneurysm treatment centers was conducted. Procedures involving the Surpass FD were conducted almost exclusively by experienced neuro-interventionalists with a combined experience of greater than 400 Pipeline FDs at two large academic comprehensive stroke centers.

Results All consecutive cases done between 1/10/2019 to $1 / 2$ / 2020 were included. There were 55 patients with 68 treated aneurysms. Procedure related complications occurred in 12 patients $(21.8 \%$ of cases) with permanent neurologic morbidity present in 11 patients (20.0\%). Mortality attributable to complications from Surpass stenting occurred in 2 patients (3.6\%). Ophthalmologic thromboembolic complications consisting of visual field compromise, ophthalmic artery thrombosis, or retinal hemorrhage occurred in 4 patients $(7.3 \%)$. Stent thrombosis occurred in 2 cases (3.6\%). Technical difficulty in device deployment resulted in 3 complications (5.5\%): confirmed distal MCA guidewire perforation; suspected distal MCA guidewire perforation as a cause of immediate post-procedural subarachnoid hemorrhage; and proximal internal carotid artery dissection resulting in ischemic stroke. In one case the Surpass device could not initially be deployed but procedure was repeated later with a proctor present. Seizures developed following the procedure in 5 patients, with focus identified on ipsilateral side in 3 cases $\&$ no definitive focus identified in 1 case $(7.3 \%$ combined attributable cases).

Conclusion Complication rates in addition to technical issues in deployment with the Surpass FD were higher than anticipated, even when performed by experienced neuro-interventionalists. The types of the complications included technical difficulty in device deployment, vascular injury, thromboembolic events and unexplained ipsilateral seizures. Large scale future registry analysis should focus on national data regarding the safety profile of Surpass.

\section{REFERENCES}

1. Wakhloo AK, et al. Surpass flow diverter in the treatment of intracranial aneurysms: a prospective multicenter study. AJNR Am J Neuroradiol 2015 Jan;36 (1):98-107.

2. Kallmes DF, et al. International retrospective study of the pipeline embolization device: a multicenter aneurysm treatment study. AJNR Am J Neuroradiol 2015 Jan;36(1):108-115.

3. Park MS, et al. Pipeline embolization device with or without adjunctive coil embolization: analysis of complications from intrePED registry. AJNR Am J Neuroradiol 2016 Jun;37(6):1127-1131.

Disclosures S. Roychowdhury: 2; C; Stryker Neurovascular. 5; C; Surpass Streamline Proctor for Stryker Neurovascular. I. Kane: None. A. Saifuddin: None. P. Khandelwal: 3; C; Honoraria speaker for American Academy of Cardiology. E. Nourallah-Zadeh: None. G. Gupta: None.

\section{0-017 REPEAT FLOW DIVERSION FOR PREVIOUSLY FAILED FLOW DIVERSION: MULTICENTER EXPERIENCE}

${ }^{1} \mathrm{M}$ Salem*, ${ }^{2} \mathrm{~A}$ Sweid, ${ }^{3} \mathrm{~A}$ Kuhn, ${ }^{4} \mathrm{~A}$ Dmytriw, ${ }^{1} \mathrm{~S}$ Gomez-Paz, ${ }^{1} \mathrm{G}$ Maragkos, ${ }^{5} \mathrm{M}$ Wagas, ${ }^{6} \mathrm{C}$ Parra-Farinas, ${ }^{7} \mathrm{~A}$ Salehani, ${ }^{8} \mathrm{~N}$ Adeeb, ${ }^{9} \mathrm{P}$ Brouwer, ${ }^{10} \mathrm{G}$ Pickett, ${ }^{11} \mathrm{M}$ Ghuman, ${ }^{11} \mathrm{~V}$ Yang, ${ }^{12} \mathrm{~A}$ Weill, ${ }^{13} \mathrm{C}$ Cognard, ${ }^{14} \mathrm{~L}$ Renieri, ${ }^{15} \mathrm{P}$ Kan, ${ }^{14} \mathrm{~N}$ Limbucci, ${ }^{4} \mathrm{~V}$ Mendes Pereira, ${ }^{7} \mathrm{M}$ Harrigan, ${ }^{3} \mathrm{~A}$ Puri, ${ }^{5} \mathrm{E}$ Levy, ${ }^{1} \mathrm{~J}$ Moore, ${ }^{1} \mathrm{C}$ Ogilvy, ${ }^{4} \mathrm{~T}$ Marotta, ${ }^{2} \mathrm{P}$ Jabbour, ${ }^{1} \mathrm{~A}$ Thomas. ${ }^{1}$ Neurosurgery, Beth Israel Deaconess Medical Center, Boston, MA; ${ }^{2}$ Neurosurgery, Thomas Jefferson University Hospitals, Philadelphia, PA; ${ }^{3}$ Neuroradiology, University of Massachusetts Medical Center, Worcester, MA; ${ }^{4}$ Neuroradiology, Toronto Western Hospital, Toronto, ON, CANADA; ${ }^{5}$ Neurosurgery, Jacobs School of Medicine and Biomedical Sciences, University at Buffalo, Buffalo, NY; ${ }^{6}$ Neurosurgery, St. Michael's Hospital, Ontario, Canada, Toronto, ON; ${ }^{7}$ Neurosurgery, University of Alabama at Birmingham, Birmingham, AL; ${ }^{8}$ Neurosurgery, Ochsner-Louisiana State University Hospital, Shreveport, LA; ${ }^{9}$ Neurosurgery, Karolinska Universitetssjukhuset, Stockholm, Stockholm, SWEDEN; ${ }^{10}$ Neurosurgery, Dalhousie University, Halifax, Nova Scotia, Canada, Halifax, NS, CANADA; " Neurosurgery, Sunnybrook Health Sciences Centre, Toronto, Canada, Toronto, ON, CANADA; ${ }^{12}$ Neurosurgery, Centre Hospitalier de I'Université de Montréal (CHUM), Montreal, Quebec, Canada, Montréal, QC, CANADA; ${ }^{13}$ Neurosurgery, University Hospital of Toulouse, Toulouse, France, Toulouse, FRANCE; ${ }^{14}$ Neuroradiology, Department of Interventional Neuroradiology, University of Florence, Florence, Italy, Florence, ITALY; ${ }^{15}$ Neurosurgery, Baylor College of Medicine, Houston, Texas, Houston, TX

\subsection{6/neurintsurg-2020-SNIS.17}

Background Aneurysmal persistence after flow-diversion (FD) occurs in $5-25 \%$ of aneurysms which might necessitate further treatment. A frequently used retreatment paradigm utilizes the deployment of another flow-diverting device (FDD) in a telescoping fashion within the existing device. There are no current data evaluating this strategy.

Methods A retrospective review of patients undergoing FD retreatment from 15 centers was performed, with inclusion criteria being repeat FD occurring for the same aneurysm at least 6 months after initial treatment, with minimum of 6 months of imaging follow-up after retreatment. Primary outcome was aneurysmal occlusion, and secondary outcomes were safety and complications. A multivariable logistic regression model was constructed to identify predictors of persistence/ occlusion after retreatment. Comparative Kaplan-Meier curves were developed to assess the effect of early (6-12 months since initial treatment) vs late retreatment ( $>12$ months since initial treatment) on the cumulative incidence of aneurysm occlusion over time. 


\begin{tabular}{llll} 
Abstract 0-017 Table 1 & & & \\
\hline Variable & OR & $95 \% \mathrm{Cl}$ & P value \\
\hline Retreatment interval & & & \\
Early (6-12 months) & 1.00 & & 0.29 \\
Late (>12 months) & 1.62 & $0.63-4.1$ & 0.29 \\
Age, 1-year increments & 1.02 & $0.978-1.06$ & 0.37 \\
Female sex & 2.72 & $0.6-8.5$ & 0.22 \\
Maximal diameter, 1-mm increments & 0.96 & $0.89-1.02$ & 0.25 \\
Smoking & 0.29 & $0.1-0.86$ & 0.026 \\
Family history of aneurysms & 0.18 & $0.04-0.78$ & 0.019 \\
Hypertension & 3.10 & $0.98-6.3$ & 0.055 \\
Fusiform morphology & 7.20 & $1.97-20.8$ & 0.003 \\
Incorporated branch into aneurysm & 2.78 & $0.98-6.8$ & 0.054 \\
\hline
\end{tabular}

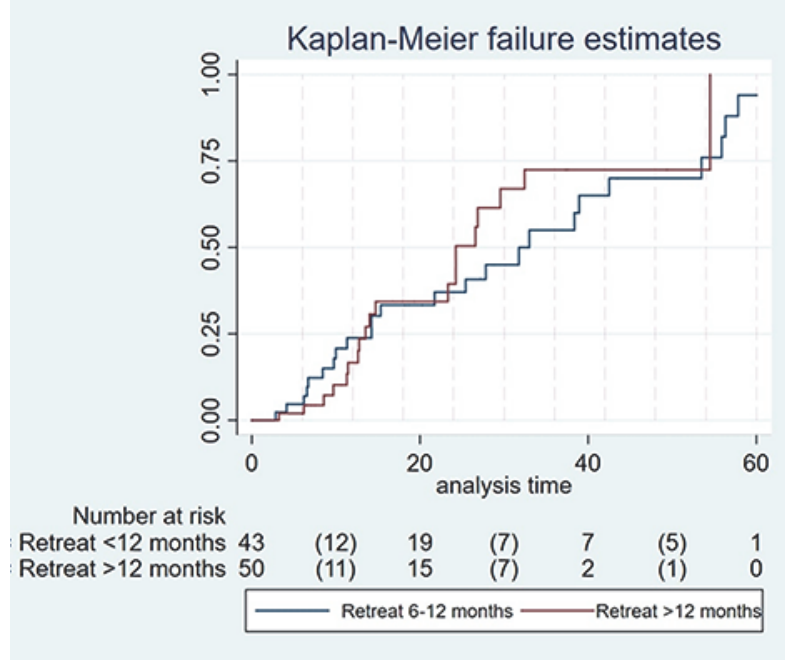

\section{Abstract 0-017 Figure 1}

Results Ninety-five patients (median age 57, 81\% females) harboring 95 aneurysms underwent 198 treatment procedures. In our cohort, $87.4 \%$ of aneurysms were unruptured; $74.7 \%$ were saccular and $79 \%$ were located in the internal carotid artery (median maximal diameter 9-mm), with 87 patients treated twice and 8 patients treated thrice. Median elapsedtime between first and second treatment was 12.2 months. Last available follow-up was performed at median of 12.8 months after retreatment, and median of 30.6 months after initial treatment, showing complete occlusion in $46.2 \%$, and near-complete occlusion (90-99\%) in $20.4 \%$ of aneurysms. No difference in ischemic complications following initial treatment and retreatment $(4.2 \%$ vs $4.2 ; \mathrm{p}>0.99)$. On multivariable logistic regression, fusiform morphology was associated with higher odds of non-occlusion after retreatment (OR 7.2; $\mathrm{p}=0.003$ ), with history of hypertension and incorporated branch into aneurysms trending toward incomplete occlusion $(p=0.055$ and $p=0.054$; respectively). Family history of aneurysms and positive smoking history were associated with higher odds of complete occlusion $(\mathrm{p}=0.019$ and $\mathrm{p}=0.026$; respectively). Kaplan-Meier curves comparative estimators showed no significant difference in time-to-occlusion between the two groups (log rank test, $\mathrm{p}=0.48$ ).

Conclusion Repeat flow diversion for persistent aneurysms is safe and effective. Fusiform morphology is the strongest predictor of aneurysmal persistence after repeat flow diversion.
Disclosures M. Salem: None. A. Sweid: None. A. Kuhn: None. A. Dmytriw: None. S. Gomez-Paz: None. G. Maragkos: None. M. Waqas: None. C. Parra-Farinas: None. A. Salehani: None. N. Adeeb: None. P. Brouwer: None. G. Pickett: None. M. Ghuman: None. V. Yang: None. A. Weill: None. C. Cognard: None. L. Renieri: None. P. Kan: None. N. Limbucci: None. V. Mendes Pereira: None. M. Harrigan: None. A. Puri: None. E. Levy: None. J. Moore: None. C. Ogilvy: None. T. Marotta: None. P. Jabbour: None. A. Thomas: None.

\section{0-018 SEIZURE PROPHYLAXIS IN UNRUPTURED ANEURYSM REPAIR: A RANDOMIZED CONTROLLED TRIAL}

B Daou* , K Holste, G Palmateer, B Thompson, C Lint, J Elenbaas, C Maher, A Pandey. Neurosurgery, University of Michigan, Ann Arbor, MI

10.1136/neurintsurg-2020-SNIS. 18

Introduction Prophylactic antiepileptic drugs (AEDs) are often prescribed for seizure prophylaxis in patients undergoing surgical treatment of unruptured intracranial aneurysms (UIAs). We aimed to evaluate the benefit of prophylactic AEDs in patients undergoing surgical repair of UIAs.

Methods We randomly assigned eligible patients undergoing surgical repair of UIAs to receive levetiracetam for seven days post-operatively or standard care alone. The primary outcome was the evaluation of seizures in the perioperative period (within 4 weeks). We also evaluated seizure occurrence throughout follow-up and assessed functional outcomes using the modified Rankin scale score (mRS).

Results 35 patients were randomized to the 'no levetiracetam' group and 41 patients were randomized to receive levetiracetam. The two study groups had similar overall baseline characteristics and the surgical complication rate was similar for both groups $(\mathrm{P}=0.8)$. One patient in the 'no levetiracetam' group had a seizure in the perioperative period versus 2 patients in the group randomized to receive levetiracetam $(2.9 \%$ vs $4.9 \%$, respectively, $\mathrm{P}=1.00)$. No patients in the 'no levetiracetam' group had any additional late seizures (mean follow-up of 20.4 months), but three patients in the levetiracetam group had late seizures during follow-up (mean followup of 19.1 months) ( $0 \%$ vs $7.3 \%, \mathrm{P}=0.2)$. mRS score of $0-2$ at 90 days and at the latest follow-up were similar between the two groups $(\mathrm{P}=1.00)$.

Conclusions Perioperative seizure prophylaxis with levetiracetam does not reduce the rate of seizures as compared to controls in patients undergoing surgical repair of UIAs.

Abstract 0-018 Table 1 Outcomes

\begin{tabular}{llll}
\hline & 'No levetiracetam' & Levetiracetam & P value \\
\hline Peri-operative seizures (within 4 weeks) & $1(2.9 \%)$ & $2(4.9 \%)$ & 1 \\
Late seizures & $0(0 \%)$ & $3(7.3 \%)$ & 0.2 \\
Any seizure & $1(2.9 \%)$ & $5(12.2 \%)$ & 0.2 \\
Median mRS at 90 days & 0 & 1 & 0.3 \\
Median mRS at the latest follow-up & 0 & 1 & 0.15 \\
mRS 0-2 at 90 days & $96 \%$ & $96 \%$ & 1 \\
mRS 0-2 at latest follow-up & $97 \%$ & $94.3 \%$ & 1 \\
\hline
\end{tabular}

